\title{
Estudio de mercado para la comercialización de los productos agrícolas, avícolas y ganaderos para el desarrollo local del cantón Santo Domingo, provincia de Santo Domingo de los Tsáchilas.
}

Market Study for the marketing of agricultural products, poultry and livestock for the local development of the canton Santo Domingo, province of Santo Domingo de los Tsáchilas.

Ramiro Enrique Guamán Chávez, MSc. ${ }^{1}$ Evelyn Karina Tinoco Díaz, MSc. ${ }^{2}$ Verónica Janneth Castro Celi.

${ }^{3}$ Guanuchi Pucha Lisseth Marisol. ${ }^{4}$

DOI: https://doi.org/10.33262/visionariodigital.v2i3.90

\section{Resumen.}

Esta investigación relacionada con el estudio de mercado para la comercialización de los productos agrícolas, avícolas y ganaderos para el desarrollo local del cantón Santo Domingo, provincia de Santo Domingo de los Tsáchilas plante como problema ¿Que incidencia tendría un estudio de mercado para la comercialización de los productos agrícolas, avícolas y ganaderos para el desarrollo local del cantón Santo Domingo?, cuyo objetivo general es elaborar un estudio de mercado para la comercialización de los productos agrícolas, avícolas y ganaderos para el desarrollo del cantón de Santo Domingo; el mismo que para su ejecución fue necesario la utilización de métodos estratégicos y/o técnicas como la encuesta, entrevista, el análisis, arrojan como resultado del diagnóstico el Plan para la implementación de una granja integral para desarrollar un ciclo óptimo de producción agrícola, avícola y ganadera del cantón Santo Domingo, para el desarrollo local y mejorar la productividad y calidad de vida de productores.

\footnotetext{
${ }^{1}$ Universidad Téc. Luis Vargas Torres, Esmeraldas. Ext. Concordia, ramiro.guaman@utelvt.edu.ec

${ }^{2}$ Universidad Téc.Luis Vargas Torres de Esmeraldas. Ext. Concordia, kaevecris@ hotmail.

${ }^{3}$ Estudiante de la carrera de Administración Públicas, verito952012@ hotmail.com.

${ }^{4}$ Estudiante de la carrera de Administración Públicas,liss.leo@ hotmail.com
} 
Palabras clave: Producción agrícola, estudio de mercado, mercado agrícola, comercio.

\begin{abstract}
.
This research related to the market study for the marketing of agricultural, poultry and livestock products for the local development of Santo Domingo canton, province of Santo Domingo de los Tsachilas, as a problem. What impact would a market study have on the commercialization of agricultural, poultry and livestock products for the local development of the Santo Domingo canton ?, whose general objective is to prepare a market study for the commercialization of agricultural, poultry and livestock products for the development of the canton of Santo Domingo; the same as for its execution it was necessary to use strategic and / or technical methods such as the survey, interview, analysis, and as a result of the diagnosis, the Plan for the implementation of an integral farm to develop an optimal cycle of agricultural production, poultry and livestock of the Santo Domingo canton, for local development and to improve the productivity and quality of life of producers.
\end{abstract}

Keywords: Agricultural production, market research, agricultural market, trade.

\title{
I. Introducción.
}

En mayo de 1994 el gobierno ecuatoriano anuncia, a través del Banco Nacional de Fomento, el inicio de un programa de apoyo dirigido a ofrecer alternativas a los pequeños productores agrícolas. El programa denominado Granjas Integrales Autosuficientes es presentado como la constitución de fincas agropecuarias altamente diversificadas con dimensiones de 1 a 10 has.

El Ecuador es un país eminentemente agrícola, el PIB Agropecuario en el año 2009 alcanzó un monto de 2.144 millones de dólares, que equivale a un aporte al PIB Total del 16,9\%; en el año 2010 se proyecta un PIB Agropecuario de 2.999 millones de dólares, que representa un aporte al PIB Total del 17,3\%; estas cifras claramente dejan ver la dinámica del sector agropecuario. (Pesca, 2015)

El Cantón de Santo Domingo es una zona netamente agrícola y ganadera pues por su ubicación geográfica, su clima, su orografía y su hidrografía son idóneas para realizar este tipo de actividades. Con la granja se busca diversificar e integrar la producción agrícola avícola y ganadera para aumentar las fuentes de ingreso y no depender exclusivamente de un producto. Así al dañarse la cosecha o caer el precio en el mercado puede recurrirse a otro producto de la granja. Esto es un seguro contra los imprevistos tan comunes en el sector agropecuario. 
Otro aspecto positivo es aumentar la variedad de productos lo cual contribuye al mejoramiento de la calidad de vida, a una mejer alimentación y no es necesario comprar aquello que puede producirse en la granja. El nivel de impacto económico, social y territorial lo mediríamos con el plan asumido en los aspectos relevantes del desarrollo como la economía y en los avances sustanciales en la calidad de vida y desarrollo territorial. Se entiende como factibilidad a la disponibilidad de recursos necesarios para cumplir con los objetivos de una actividad o proyecto. El proyecto de factibilidad consiste en un documento escrito que sirve como herramienta y guía de un proceso lógico, progresivo, realista, coherente y orientado a la acción futura para tomar la decisión de asignar recursos hacia un objetivo determinado. (Berghe, 2014)

Es un plan de vida para las familias asentadas en el campo, que además de asegurar una alimentación abundante y rica en proteínas, vitaminas y minerales le enseña a cada uno de sus integrantes a vivir con armonía con la naturaleza, perseverando y disfrutando el medio que lo rodea, respirando aire puro. (kuhne, 2011)

Adicionalmente la granja integral autosuficiente estimula el uso de tecnologías apropiadas a bajo costo como el empleo de la energía eólica energía solar y producción de gas metano que manejadas de forma adecuada contribuyen al bienestar de la familia rural, lo cual facilita en corto tiempo alcanzar los niveles de autosuficiencia y sostenibilidad deseados. (Instituto nacional de investigaciones, 2012)

Promover una agricultura sana sin uso de fertilizantes, plaguicidas, herbicidas y cualquier tipo de producto químico, así como no utilizar costosos concentrados para alimentación animal. Por el contario un fundamento del sistema es el reciclaje de todos los elementos de la granja. (Ariadna, 2011)

Para realizar un publicación de mercado Según (Berghe, 2014) "El estudio de mercado utiliza una serie de técnicas útiles para obtener información del medio que rodea al proyecto, que le permita pronosticar las tendencias futuras de su comportamiento". Con la granja integral se buscar diversificar e integrar la producción agraria para aumentar la fuente de ingreso y no depender exclusivamente de un producto. Aplicando la investigación del mercado hablamos de una de las herramientas más útiles para conocer la situación del entorno que rodea a la organización ya que se analiza todos los datos relacionados con los problemas en la comercialización de bienes y servicios. Esta investigación es aplicable a cualquier fase de la comercialización después se determina el área para la instalación de cada una de las zonas de la granja teniendo en cuenta que cuando los suelos son fértiles es posible utilizar menores extensiones de tierra y cuando estos no lo son se requiere un área mayor. (Luis M. , 2012)

Para iniciar la investigación de mercados se debe empezar por la definición del problema, una vez que el problema está bien definido se puede seguir con el desarrollo de la investigación. Las tareas que implican la formulación del problema de mercados, 
incluyen el análisis con los ejecutivos clave en la toma de decisiones, así como la auditoria del problema, entrevistas con expertos de la industria, análisis de datos secundarios e investigación cualitativa. (Luis R. , 2011)

El diseño de la investigación es una estructura o plano que sirve para llevar a cabo el proyecto de investigación de mercado, especifica los detalles de los procedimientos necesarios para obtener la información requerida y estructurar o resolver los interrogantes de la investigación, de manera que establece las bases para que el proyecto se ejecute de una manera efectiva y eficiente. Debemos tener en cuenta una economía conjunta con la ecología; Con la granja integral se busca diversificar e integrar la producción agraria para aumentar las fuentes de ingreso y no depender exclusivamente de un producto, así al dañarse una cosecha o caer el precio del mercado puede recurrirse a otro producto del rancho. (Luis R. , 2011)

Tendremos como resultados enriquecer el suelo con humus que resulta de la descomposición de la materia orgánica, lo que le da mayor grado de fertilidad y aumento en la capacidad de retención de humedad.

Asimismo, se debe tomar algunas medidas preventivas controlando las plagas mediante insecticidas y reciclar todos los desperdicios de la granja. (Pesca, 2015), La granja debe verse como un conjunto de elementos en el que se integran la seguridad alimentaria y económica, el agua, el suelo, la producción vegetal y animal respetando la naturaleza. (/FAO, 2015)

El primer paso para montar la granja integral autosuficiente es determinar el área para la instalación de cada una de las zonas de la granja. El siguiente paso es construir la vivienda para la familia esta debe ser cómoda e higiénica. Hay que resaltar dos factores prioritarios en el momento de planificar la granja y que esta nos de ganancias; las condiciones agroecológicas y las condiciones de mercado, elaborar un mapa del terreno distribuyendo las áreas destinadas para frutales, hortalizas, pastos de corte. (FAO, 2014)

Una vez definidas las líneas de producción de la granja y su demanda en el mercado, es importante incorporar cultivos considerando su ciclo estas pueden ser ideas para la granja integral sin embargo deben adaptarse de acuerdo con la experiencia del agricultor y con la superficie y los recursos disponibles. (Lu Flora, 2013) De acuerdo con la organización de los recursos humanos es útil compartir los conocimientos que adquieran con sus colaboradores e impulsarlos para que aprendan más sobre los cultivos y todos los asuntos referentes a la producción. La rentabilidad viene en la buena utilización de recursos como una de las herramientas más importantes que dispone la administración empresarial, es la base para la determinación de la eficiencia del proceso de producción que se analiza.

\section{Metodos y materiales.}


La técnica de recolección de datos que se empleará en esta investigación la observación, con un método descriptivo. No obstante, se emplean una amplia gama de medios, procedentes, en muchos casos, de otras disciplinas, como la sociología o psicología, en los que incluye el análisis cuantitativo de datos estadísticos o las encuestas y entrevistas. Los temas objeto de estudio son muy variados. Sucediendo con la comercialización de productos agrícolas, avícolas y ganaderos del cantón Santo Domingo, provincia de Santo Domingo. Además, se utilizó esta técnica al inicio, en el proceso y al final de la investigación propuesta.

Se aplicó la encuesta a los productores agrícolas, avícolas y ganaderos del cantón Santo Domingo, provincia de Santo Domingo. Se diseñaron Guías de Observación para rescatar problemas referidos a la investigación. Las informaciones obtenidas por las técnicas citadas se ordenaron, clasificaron, resumieron, presentaron y graficaron en función a los objetivos de la investigación. Los datos obtenidos fueron interpretados según los objetivos de a investigación generándose el contraste de las preguntas directrices y entre las principales técnicas de análisis que se emplearon en la presente investigación tenemos la codificación, tabulación y técnicas estadísticas.

\section{Análisis e interpretación de los resultados.}

Tabla 1. ¿Existen muchos productores agrícolas, avícolas y ganaderos en el Santo Domingo, provincia de Santo Domingo?

\begin{tabular}{ccc}
\hline Opción & F & \% \\
\hline SI & 78 & 90 \\
NO & 9 & 10 \\
TOTAL & 87 & 100 \\
\hline
\end{tabular}

Elaborado por: Grupo de Investigadores

Tabulada la primera pregunta con relación a si muchos productores agrícolas, avícolas y ganaderos del cantón Santo Domingo, provincia de Santo Domingo se obtuvo las siguientes respuestas; el $90 \%$ que equivale a 78 personas encuestadas respondieron que si existen muchos productores, mientras que el $10 \%$ que equivale a 9 personas encuestadas dijeron que no.

Tabla 2. ¿En el cantón Santo Domingo existe producción agrícola, avícola y ganadera todo el año?

\begin{tabular}{ccc}
\hline OPCION & F & \% \\
\hline SI & 75 & 86
\end{tabular}




$\begin{array}{ccc}\text { NO } & 12 & 14 \\ \text { TOTAL } & 87 & 100\end{array}$

Elaborado por: Grupo de Investigadores

De acuerdo a las coordinaciones efectuadas, se optó por encuestar a un total de 87 personas involucradas en el ámbito de la presente investigación de los cuales 75 de ellos manifestaron que, SI y mientras que 12 contestaron que no, tal como lo muestra en cuadro Mediante los resultados de este cuadro, muestra que el $86 \%$ opinaron que existe producción todo el año lo cual permite a los diferentes productores poder comercializar la producción agrícola, avícola y ganadera en la región y fuera de ella, mientras que el $14 \%$ no existe producción.

Tabla 3. ¿Qué técnicas utilizan para el mercadeo de productos agrícolas, avícolas y ganaderos del cantón Santo Domingo, provincia de Santo Domingo?

\begin{tabular}{lcc}
\hline Técnicas & F & \% \\
\hline Compra -venta & 38 & 41 \\
Promociones & 11 & 13 \\
Propaganda & 20 & 23 \\
Venta itinerante & 12 & 14 \\
Otros & 8 & 9 \\
Total & $\mathbf{8 7}$ & $\mathbf{1 0 0}$ \\
\hline
\end{tabular}

Elaborado por: Grupo de Investigadores

A un total de 87 personas involucradas en el ámbito de la presente investigación, de los cuales 36 de ellos manifestaron que compra y venta, 11 que promociones, 20 en propagandas, 12 aseguran que venta itinerante y mientras que 8 contestaron que otras técnicas, tal como lo muestra el cuadro, al observar los resultados arrojados en este cuadro, se nota que el $41 \%$ de los productos utilizan compra venta como técnica de mercadeo $13 \%$ de los productos utilizan promociones, $23 \%$ de los productores utilizan propaganda $14 \%$ de los productores utilizan la venta itinerante, $9 \%$ de los productores utilizan otra técnica del mercadeo.

Tabla 4. ¿A quién usted le vende los productos agrícolas, avícolas y ganaderos del cantón Santo Domingo?

\begin{tabular}{lll}
\hline Destino final & F & \% \\
\hline Camioneros & 28 & 32 \\
Abastos & 4 & 5 \\
Mercado & 8 & 9 \\
Mercado de coche & 39 & 45 \\
Consumidor & 8 & 9 \\
Total & 87 & 100 \\
\hline
\end{tabular}


Elaborado por: Grupo de Investigadores

Del personal involucrados en el ámbito de la presente investigación; de 28 de ellos manifestaron que venden a camioneros, 4 que, a centros de abastos, 8 en el mercado, 39 aseguran que a coches de mercado y mientras que 8 contestaron que, al consumidor final, los resultados evidencian con claridad que el $32 \%$ de los productores les venden sus productos a los abastos, el $9 \%$ de los productores les venden sus productos a los mercados, $45 \%$ de los productores le venden sus productos al mercado de coche, $9 \%$ de sus productores les vende sus productos a los consumidores.

Tabla 5. ¿Qué cantidad en (unidades) de productos agrícolas, avícolas y ganaderos del cantón Santo Domingo, provincia de Santo Domingo vende usted?

\begin{tabular}{lll}
\hline Destino final & F & $\%$ \\
\hline $\mathbf{0 - 5}$ & 0 & 0 \\
$\mathbf{0 5 - \text { oct }}$ & 4 & 5 \\
oct-15 & 8 & 9 \\
$\mathbf{1 5 - 2 0}$ & 12 & 14 \\
Otros (20 y más) & 63 & 72 \\
Total & 87 & 100 \\
\hline
\end{tabular}

Elaborado por: Grupo de Investigadores

De 4 personas que venden entre 5-10 unidades, 8 que, entre 10-15 unidades, 12 que entre 15 - 20 unidades, y mientras que 63 contestaron que 20 o más unidades, Tal como lo muestra los resultados del cuadro anterior indicaron que el 5\% de los productores venden entre 5 y 10 unidades, la cantidad de sus productos, el $9 \%$ de los productores venden Entre 10- 15 unidades. Sus productos, el 14\% de productores venden entre 15 - 20 unidades, sus productos y el $72 \%$ de los productores venden más de 20 unidades. Sus productos. Esto se debe a que esté $72 \%$ se dedican única y exclusivamente a la producción agrícola y ganadera, Mientras que el porcentaje restante son personas que se dedican a otro tipo de actividad.

Tabla 6. ¿Cuál es la frecuencia de venta de los productos agrícolas, avícolas y ganaderos del cantón Santo Domingo, provincia de Santo Domingo?

\begin{tabular}{lcc}
\hline Frecuencia & F & \% \\
\hline Diario & 4 & 5 \\
Semanal (lunes - viernes) & 70 & 81 \\
Fines de semana (sábado y domingo) & 8 & 9 \\
Quincenal & 5 & 5 \\
Mensual & 0 & 0 \\
Otros & 0 & 0 \\
Total & 87 & 100 \\
\hline
\end{tabular}


Elaborado por: Grupo de Investigadores.

De 87 personas involucradas en el ámbito de la presente investigación; de los cuales 4 de ellos manifestaron que venden a diario, 70 que semanal de lunes a viernes, cinco contestaron que quincenalmente, de los resultados que arrojó el cuadro anterior se evidenció que el 5\% de los productores venden sus productos diariamente, el $81 \%$ de los productores venden sus productos semanales, el 9\% de los productores venden sus productos los fines de semana, el 5\% de los productores venden sus productos quincenalmente.

Tabla 7. ¿A qué precio (dólar) usted vende los productos agrícolas, avícolas y ganaderos del cantón Santo Domingo, provincia de Santo Domingo?

\begin{tabular}{lcc}
\hline Frecuencia & F & \% \\
\hline 10 & 4 & 5 \\
12 & 8 & 9 \\
14 & 17 & 19 \\
16 & 23 & 27 \\
20 & 31 & 35 \\
Otros & 5 & 5 \\
Total & $\mathbf{8 7}$ & $\mathbf{1 0 0}$ \\
\hline
\end{tabular}

Elaborado por: Grupo de Investigadores.

Con un total de 87 personas involucradas en el ámbito de la presente investigación; de los cuales 4 de ellos manifestaron que venden a \$10 USD, 8 que a \$12 USD, 17 que a \$14 USD, 23 que a \$16 USD, 31 que a \$20 USD y mientras que cinco contestaron que otros valores, al observar los resultados arrojados en este cuadro, se nota que el 5\% de los productores venden sus productos a \$10 USD, 9 por ciento de los productores venden sus productos a \$12 USD, 19\% de los productores venden sus productos a \$14 USD, 27\% de los productores venden sus productos a \$16 USD, 35\% de los productores vende sus productos a \$20 USD, y el 5\% de los productores venden sus productos a más de $\$ 20$ USD, la variación de los precios se debe a que a mayor cantidad de venta, menor es el precio, además también varía el precio dependiendo de la calidad del producto y del arreglo con los comerciantes.

Tabla 8. ¿Dónde almacena sus productos agrícolas, avícolas y ganaderos del cantón Santo Domingo, provincia de Santo Domingo?

\begin{tabular}{lcc}
\hline Lugar & F & $\%$ \\
\hline Terreno & 16 & 18 \\
Casa & 67 & 77 \\
Otros & 4 & 5 \\
Total & 87 & 100 \\
\hline
\end{tabular}


Elaborado por: Grupo de Investigadores.

De la siguiente pregunta 67 que lleva a su casa y almacena, mientras que 4 contestaron que otros lugares, de los resultados arrojados de este cuadro se pudo observar que el $18 \%$ de los productores almacenan sus productos en el terreno, $77 \%$ de los productores almacena sus productos en la casa, 5\% de los productores almacenan sus productos en otros lugares. Esto se debe a que no tiene un lugar fijo que reúna las condiciones necesarias de almacenamiento, y por el alto costo de algunos de estos lugares.

Tabla 9. ¿Existe un centro de acopio de los productos agrícolas, avícolas y ganaderos del cantón Santo Domingo, provincia de Santo Domingo?

\begin{tabular}{ccc}
\hline Opción & F & \% \\
\hline $\mathrm{Si}$ & 0 & 0 \\
No & 87 & 100 \\
Total & 87 & 100 \\
\hline
\end{tabular}

Elaborado por: Grupo de Investigadores.

Según los resultados arrojados de este cuadro, se puede observar que el $100 \%$ de la muestra con 87 personas opinaron no existe un centro de acopio en donde los productores puedan almacenar sus productos y así poderlas vender a un mejor precio y obtener mayor ganancia

Tabla 10. ¿Considera que se debe realizar un estudio de mercado para la comercialización de los productos agrícolas, avícolas y ganaderos?

\begin{tabular}{ccc}
\hline Opción & F & \% \\
\hline $\mathrm{Si}$ & 67 & 77 \\
No & 20 & 23 \\
Total & 87 & 100 \\
\hline
\end{tabular}

Elaborado por: Grupo de Investigadores.

Se optó por encuestar a un total de 87 personas involucradas en el ámbito de la presente investigación; de los cuales 67 de ellos manifestaron que si es necesario realizar un estudio de mercado para la comercialización de los productos agrícolas, avícolas y ganaderos. Mientras que 20 opinaron que no, tal como lo muestra el cuadro número 10, Según los resultados arrojados en este cuadro, el $77 \%$ de los productores considera que se debe realizar un estudio de mercado para la comercialización de los productos agrícolas, avícolas y ganaderos, $23 \%$ considera que no es necesario realizar un estudio de 
mercado para la comercialización de los productos agrícolas, avícolas y ganaderos avícolas y ganaderos.

Tabla 11. ¿Está usted interesado en participar en las actividades que se organizan para elaborar un estudio de mercado para la comercialización de los productos agrícolas, avícolas y ganaderos?

\begin{tabular}{ccc}
\hline Opción & F & \% \\
\hline Si & 63 & 72 \\
No & 24 & 28 \\
Total & 87 & 100 \\
\hline
\end{tabular}

Elaborado por: Grupo de Investigadores

De las personas encuestadas 63 de ellos manifestaron que sí participarían de las actividades que se organizan para elaborar un estudio de mercado para la comercialización de los productos agrícolas, avícolas y ganaderos, mientras que 24 opinaron que no, se puede observar que los resultados arrojados por este cuadro, el $72 \%$ de los productores estarían dispuestos a participar en las diferentes actividades para conocer las funciones que cumple un estudio de mercado para la comercialización de los productos agrícolas, avícolas y ganaderos, y el $28 \%$ no tienen mucho interés en participar porque no quieren conocer en qué consiste un estudio de mercado para la comercialización de los productos agrícolas, avícolas y ganaderos.

Tabla 12. ¿Al realizar un estudio de mercado para la comercialización de los productos agrícolas, avícolas y ganaderos del cantón Santo Domingo, Qué beneficios obtendría usted?

\begin{tabular}{lll}
\hline Opción & F & \% \\
\hline Obtener mayor ganancia & 12 & 14 \\
$\begin{array}{l}\text { Establecer el precio a los diferentes } \\
\text { compradores }\end{array}$ & 12 & 14 \\
Mejorar su nivel de vida & 14 & 16 \\
Aumentar las ventas & 41 & 47 \\
Almacenar su producto & 8 & 9 \\
Total & $\mathbf{8 7}$ & $\mathbf{1 0 0}$ \\
\hline
\end{tabular}

Elaborado por: Grupo de Investigadores

El total de las personas encuestadas 12 de ellos manifestaron que obtienen mayor ganancia, 12 que se establece el precio de los diferentes compradores, 14 que mejoran su nivel de vida, 41 aseguran que aumenta la venta y mientras que 8 contestaron que sólo para almacenar el producto, tal como lo muestra el cuadro número 12, arrojó los siguientes resultados, 14\% de los productores obtendría 
mayor ganancia al realizar un estudio de mercado para la comercialización de los productos agrícolas, avícolas y ganaderos, $14 \%$ de los productores establece el precio de los diferentes compradores, $16 \%$ de los productores mejorará su nivel de vida, $47 \%$ de los productores aumentará su venta, $9 \%$ de los productores va a almacenar su producto, todos estos beneficios serían muy importantes para los productores entre ellos el más resaltante es el aumento de las ventas; debe ser porque al realizar un estudio de mercado para la comercialización de los productos agrícolas, avícolas y ganaderos, todos se organizarían para promover el desarrollo de su sector y los clasificarían y los venderían de acuerdo a su calidad.

\section{Conclusiones.}

- El estudio de Mercado permitió determinar que en el Cantón Santo Domingo una gran mayoría de las personas son productores agrícolas, avícolas y ganaderos, además existe producción todo el año. Los productores utilizan más la técnica de compra y venta debido a que es la que ha dado mayor resultado en cuento al Mercado de productos, agrícolas, avícolas y ganaderos.

- Es necesario a realizar un estudio de mercado para la comercialización de los productos agrícolas, avícolas y ganaderos, en el mayor porcentaje les interesa y al mismo tiempo es necesario para la correcta comercialización de sus productos.

- Los productores estarían dispuestos a participar en las diferentes actividades para conocer las funciones que cumple un estudio de mercado para la comercialización de los productos agrícolas, avícolas y ganaderos. Así se organizarían para promover el desarrollo de su sector y los clasificarían y los venderían de acuerdo a su calidad.

\section{Referencias bibliográficas.}

- /FAO, O. (2015). Perspectivas agricolas 2015. Paris.

- Ariadna, B. (2011). Agricultura Ecologica i sostenibilitat. Barcelona: UOC.

- Berghe, V. d. (2014). Tratados de libre comercio retos y oportunidades. Bogota: Ecoe.

- Domínguez , J., \& Benedicto, M. (10 de enero de 2010). Análisis EconómicoFinanciero. Obtenido de Creative Commons Reconocimiento, Nocomercial: http://api.eoi.es/api_v1_dev.php/fedora/asset/eoi:67125/componente67150.pdf

- FAO. (2014). La alimentacion y la agricultura en America Latina y el Caribe. Santiago. 
- Instituto nacional de investigaciones, f. y. (2012). Revista Mexicana de ciencias agricolas. Inifap, 199.

- Jiménez , A. (20 de diciembre de 2017). Analisis y Planificación Financiera. Obtenido de diarium: http://diarium.usal.es/ajimenez/analisis-y-planificacionfinanciera/

- kuhne, B. (2011). Cuidado de cultivos biologicos al aire libre, sanidad vegetal en el merco de la agricultura. Italia.

- Lu Flora, S. M. (2013). Integracion al mercado y salud indigena en el nororiente Ecuatoriano. Quito: Abya-yala.

- Luis, M. (2012). Avances de la investigacion Agronomica II. Bogota.

- Luis, R. (2011). Enfoques de investigacion sostenible Ecologista y productivista. Inifap, 25.

- Pesca, M. d. (2015). La politica agropecuaria ecuatoriana hacia el desarrollo territorial rural sostenible 2015-2025. Quito.

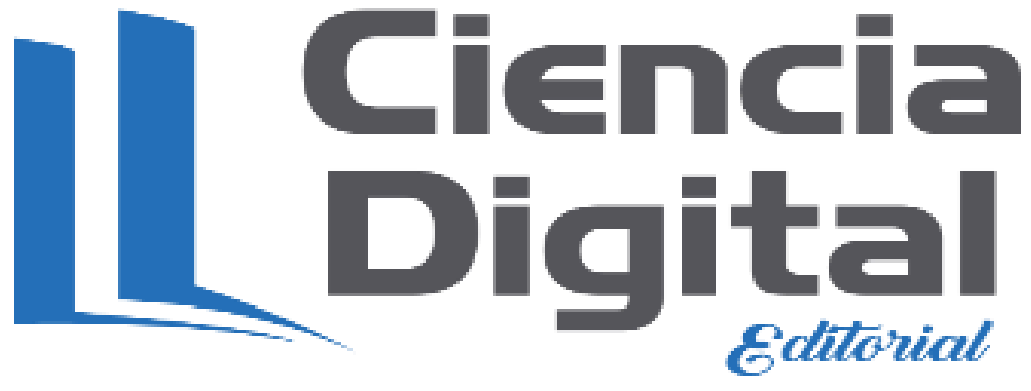


Para citar el artículo indexado.

Guamán R., Tinoco E., Castro V \& Guanuchi L. (2018). Estudio de mercado para la comercialización de los productos agrícolas, avícolas y ganaderos para el desarrollo local del Cantón Santo Domingo, provincia de Santo Domingo de los Tsáchilas. Revista electrónica Visionario Digital 1(1), 61-73. Recuperado desde: http://cienciadigital.org/revistacienciadigital2/index.php/VisionarioDigital/article/view/90/ $\underline{84}$

\section{Ciencia Ligital}

El artículo que se publica es de exclusiva responsabilidad de los autores y no necesariamente reflejan el pensamiento de la Revista Ciencia Digital.

El articulo queda en propiedad de la revista y, por tanto, su publicación parcial y/o total en otro medio tiene que ser autorizado por el director de la Revista Ciencia Digital 